Johan M. Kros • Willem C. D. Schouten

Patrick J. A. Janssen - Theodorus H. van der Kwast

Proliferation of gemistocytic cells

\title{
and glial fibrillary acidic protein (GFAP)-positive oligodendroglial cells in gliomas: a MIB-1/GFAP double labeling study
}

Received: 16 March 1995 / Revised: 24 May 1995 / Revised, accepted: 21 August 1995

\begin{abstract}
Large gemistocytic cells are well-known elements of glial tumors. Recently, miniature gemistocytic cells and neoplastic glial fibrillary acidic protein (GFAP)positive oligodendroglial cells, which are regularly seen in oligodendrogliomas, have been termed "transitional cells". The proliferative activity of the gemistocytic cell types and the GFAP-positive (gliofibrillary) oligodendrocytes was determined in eight astrocytomas, seven gemistocytic astrocytomas, eight glioblastomas, two monstrocellular glioblastomas, seven oligodendrogliomas and three mixed oligo-astrocytomas by immunohistochemical staining of the proliferation marker MIB-1 in combination with immunostaining for GFAP. Both large gemistocytic cells and the transitional cells showed cytoplasmic GFAPpositive staining. Neither in the classic gemistocytes nor in the minigemistocytes nuclear immunostaining for the MIB-1 antibody was observed. In contrast, MIB-1 staining was seen in the gliofibrillary oligodendrocytes. It is concluded that both large and miniature gemistocytic cell types contrast with gliofibrillary oligodendrocytes by their inability to proliferate.
\end{abstract}

Key words Glioma - Glial fibrillary acidic protein · Ki-67 · Proliferation · Cell kinetics

Introduction

In 1904 Franz Nissl [17] described a subset of astrocytic cells which were encountered under pathological conditions and were characterized by voluminous, homogeneous, milk-like cytoplasm with plump cell processes and an eccentric nucleus. These cells were referred to as gemästete Glia (fattened glia). In the English language

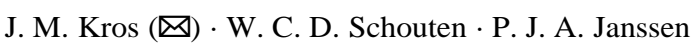

T. H. van der Kwast

Department of Pathology, Neuropathology Laboratory,

University Hospital Rotterdam-Dijkzigt, Dr. Molewaterplein 40,

3015 GD Rotterdam, The Netherlands

Tel.: 31-10-408-7905; Fax: 31-10-436-6660 this term was translated in "gemistocytes". These cells were not only encountered in reactive processes, but also in glial tumors. Moreover, some gliomas seemed to be entirely composed of these cells (gemistocytic astrocytomas or gemistocytomas). Gemistocytes were also regularly seen as a minor constituent of oligodendrogliomas. Smaller gemistocytic cells that were found in oligodendroglial tumors in particular were reappraised after the introduction of the immunohistochemical stain for glial fibrillary acidic protein (GFAP) [7, 16, 26, 27], and were lumped together with gliofibrillary (or GFAP-positive) oligodendrocytes (GFOCs) and called transitional cells [7]. Interrelationships between the transitional cells and the classic gemistocytes have been suggested based on immunological and ultrastructural similarities [7, 11, 12].

It has been shown that the presence of classic gemistocytes in astrocytomas and oligodendrogliomas is a prognostically ominous sign $[5,10,13,22]$. Although this finding might suggest high proliferation of gemistocytes, in vivo thymidine incorporation studies have shown that gemistocytes are quiescent cells $[8,9]$. The proliferative activity of the transitional cells has not been studied previously.

The aim of the present study was to investigate whether classic gemistocytes and transitional cells belong to the proliferative cell pool in gliomas. To this end, antibodies against the proliferation marker MIB-1 and GFAP were simultaneously used on formalin-fixed paraffin-embedded material of 35 glial neoplasms in which these GFAP-positive cells were seen.

\section{Materials and methods}

Clinical data

The formalin-fixed paraffin-embedded tissue samples of 15 astrocytomas (7 of which were gemistocytic astrocytomas), 7 oligodendrogliomas, 3 mixed astro-oligodendrogliomas and 10 glioblastomas (including 2 monstrocellular glioblastomas) were taken from the files of the Department of Pathology of the University Hospital Rotterdam-Dijkzigt (Rotterdam, The Netherlands) (Table 1). In addition, two cases of reactive gliosis were retrieved. No stereotactic biopsy material was included in this study. 
Table 1 Summary of cases investigated. The MIB-1 range is given as $\%$ positive nuclei/5 high-power fields. [ 0 absent, + small numbers in some fields, ++ intermediate numbers, +++ large numbers seen in many fields, gem (large) gemistocytes, mini minigemistocytes, GFOC gliofibrillary oligodendrocyte, mons monstrous cells, gem. astro gemistocytic astrocytoma, $\mathrm{gbm}$ glioblastoma]
Despite large regional differences in percentage of positive nuclei, the MIB-1 labeling index roughly correlated with tumor grades (numbers too small for statistics) a [3] b [24]

\begin{tabular}{|c|c|c|c|c|c|c|c|}
\hline & $\begin{array}{l}\text { Grade } \\
\text { (WHO) }\end{array}$ & $\begin{array}{l}\text { Grade } \\
\text { Daumas- } \\
\text { Duport }^{\mathrm{a}} \text { ) }\end{array}$ & $\begin{array}{l}\text { MIB-1 } \\
\text { range }\end{array}$ & gem & $\operatorname{mini}$ & GFOC & mons \\
\hline 1. Astrocytoma & 2 & 2 & $0-5$ & 0 & 0 & 0 & 0 \\
\hline 2. Gem. astro & 3 & 2 & $5-20$ & +++ & + & 0 & 0 \\
\hline 3- 8. Gem. astro & 3 & 3 & $5-40$ & +++ & ++ & 0 & 0 \\
\hline 9. Astrocytoma & 3 & 3 & $5-10$ & 0 & 0 & 0 & 0 \\
\hline 10. Astrocytoma & 3 & 3 & $5-10$ & 0 & + & 0 & 0 \\
\hline 11. Astrocytoma & 3 & 3 & $5-20$ & + & + & 0 & 0 \\
\hline 12. Astrocytoma & 3 & 3 & $5-25$ & + & 0 & 0 & 0 \\
\hline 13. Astrocytoma & 3 & 3 & $5-30$ & + & ++ & 0 & 0 \\
\hline 14. Astrocytoma & 3 & 3 & $5-30$ & + & + & 0 & 0 \\
\hline 15. Astrocytoma & 4 & 4 & $20-40$ & ++ & ++ & 0 & 0 \\
\hline 16-18. gbm & & & $5-50$ & ++ & 0 & 0 & 0 \\
\hline 19-20. gbm & & & $5-45$ & ++ & + & 0 & ++ \\
\hline 21-25. gbm & & & $40-50$ & 0 & 0 & 0 & 0 \\
\hline 26. Oligo-astrocytoma & 2 & 2 & $10-30$ & + & + & + & 0 \\
\hline 27. Oligo-astrocytoma & 2 & 2 & $10-40$ & + & ++ & + & 0 \\
\hline \multirow[t]{2}{*}{ 28. Oligo-astrocytoma } & 3 & 3 & $20-50$ & ++ & + & 0 & 0 \\
\hline & $\begin{array}{l}\text { Grade } \\
\text { (WHO) }\end{array}$ & $\begin{array}{l}\text { Grade } \\
\left(\text { Smith }^{b}\right)\end{array}$ & & & & & \\
\hline 29. Oligodendroglioma & 2 & 2 & $0-5$ & 0 & 0 & 0 & 0 \\
\hline 30. Oligodendroglioma & 2 & 2 & $0-5$ & + & +++ & ++ & 0 \\
\hline 31. Oligodendroglioma & 3 & 3 & $5-10$ & + & +++ & + & 0 \\
\hline 32. Oligodendroglioma & 3 & 3 & $10-20$ & 0 & + & ++ & 0 \\
\hline 33. Oligodendroglioma & 3 & 3 & $10-30$ & 0 & 0 & 0 & 0 \\
\hline 34. Oligodendroglioma & 3 & 3 & $20-40$ & + & + & + & 0 \\
\hline 35. Oligodendroglioma & 3 & 3 & $20-40$ & + & + & + & 0 \\
\hline
\end{tabular}

Grading of the astrocytomas and mixed gliomas was done according to the WHO guidelines, and to the St.Anne-Mayo scheme for astrocytic neoplasms [3]. The oligodendrogliomas were graded according to the WHO guidelines and to the recently developed scheme by Smith et al. [24]. The ages of the patients at surgery ranged between 22 and 76 years. All biopsies were performed before additional radiotherapy was given. None of the patients had been treated with chemotherapy prior to biopsy.

\section{GFAP/MIB-1 double staining}

Paraffin sections ( $5 \mu \mathrm{m}$ thick) were cut and mounted on 3-aminopropyltriethoxisilane-coated slides (Sigma, St. Louis, Mo.). After deparaffinization and rehydration, endogenous peroxidase was blocked with $3 \% \mathrm{H}_{2} \mathrm{O}_{2}$ in methanol. The sections were preincubated with normal goat serum (Dako, The Netherlands), diluted 1:10 in bovine serum albumin (BSA) [5\% in phosphate-buffered

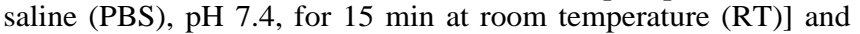
subsequently with anti-GFAP antibody (Dako, Copenhagen, Denmark; rabbit polyclonal 1:200 in BSA 5\%) for $1 \mathrm{~h}$ at RT. After rinsing in PBS, the sections were incubated with biotinylated goat anti-rabbit antibody (Dako, The Netherlands), diluted 1:400 in PBS, for 30 min, streptavidin-biotin-alkaline phosphatase complex (Dako, The Netherlands) for $30 \mathrm{~min}$ and substrate for alkaline phosphatase (Vector Kit). The sections were rinsed three times in PBS between each incubation step.

For MIB-1 staining an antigen retrieval procedure [23] was necessary. The reaction was optimized by placing the sections in a plastic box containing $200 \mathrm{ml} 0.01 \mathrm{M}$ citric acid monohydrate (Merck, Germany) adjusted to $\mathrm{pH} 6.0$ with $\mathrm{NaOH}$, and heating in a microwave oven (Miele M696) for $3 \times 5 \mathrm{~min}$ at $700 \mathrm{~W}$. After preincubation $(15 \mathrm{~min})$ with normal goat serum, diluted $1: 10$ in
Fig. 1 Gemistocytic astrocytoma grade 3 (case 2). The large gemistocytic cells are invariably negative for MIB-1, while nuclei of interspersed neoplastic glial cells are positive. Glial fibrillary acidic protein (GFAP)/MIB-1 double labeling, $\times 150$

Fig. 2 High magnification of a large (classic) MIB-1-negative gemistocytic cell in a gemistocytic astrocytoma (case 2). GFAP/ MIB-1 double labeling, $\times 1,000$

Fig. 3 Astrocytoma grade 4 (case 15) stained for GFAP and MIB1. MIB-1-immunolabeled nuclei (brown) are found in the anaplastic parts, while only sporadically MIB-1-positive nuclei are seen in the GFAP-rich (red) gemistocytic parts. GFAP/MIB-1 double labeling, $\times 150$

Fig. 4 Astrocytoma grade 3 (case 11) stained for GFAP and MIB1. Gemistocytic cells remain negative for MIB-1 immunostaining. GFAP/MIB-1 double labeling, $\times 150$

Fig.5 Oligodendroglioma grade 2 (case 30) stained for GFAP and MIB-1. Whereas some MIB-1-positive nuclei of neoplastic oligodendrocytes are observed, no immunoreactivity of nuclei of gemistocytic cells is seen. GFAP/MIB-1 double labeling, $\times 150$

Fig. 6 The term "minigemistocytoma" was applied to oligodendrogliomas that are almost entirely composed of minigemistocytes [7]. None of the minigemistocytes were immunopositive for MIB1 (case 31). GFAP/MIB-1 double labeling, $\times 150$

Fig. 7 Gliofibrillary oligodendrocytes (GFOCs) and minigemistocytes in an oligodendroglioma (case 31). The GFOCs have a small GFAP-positive rim around their nuclei (arrows). The minigemistocytes are characterized by a more voluminous GFAP-positive cytoplasm. Although the former occasionally show MIB-1 positivity, the minigemistocytes invariably were immunonegative for MIB-1. GFAP/MIB-1 double labeling, $\times 1,000$ 

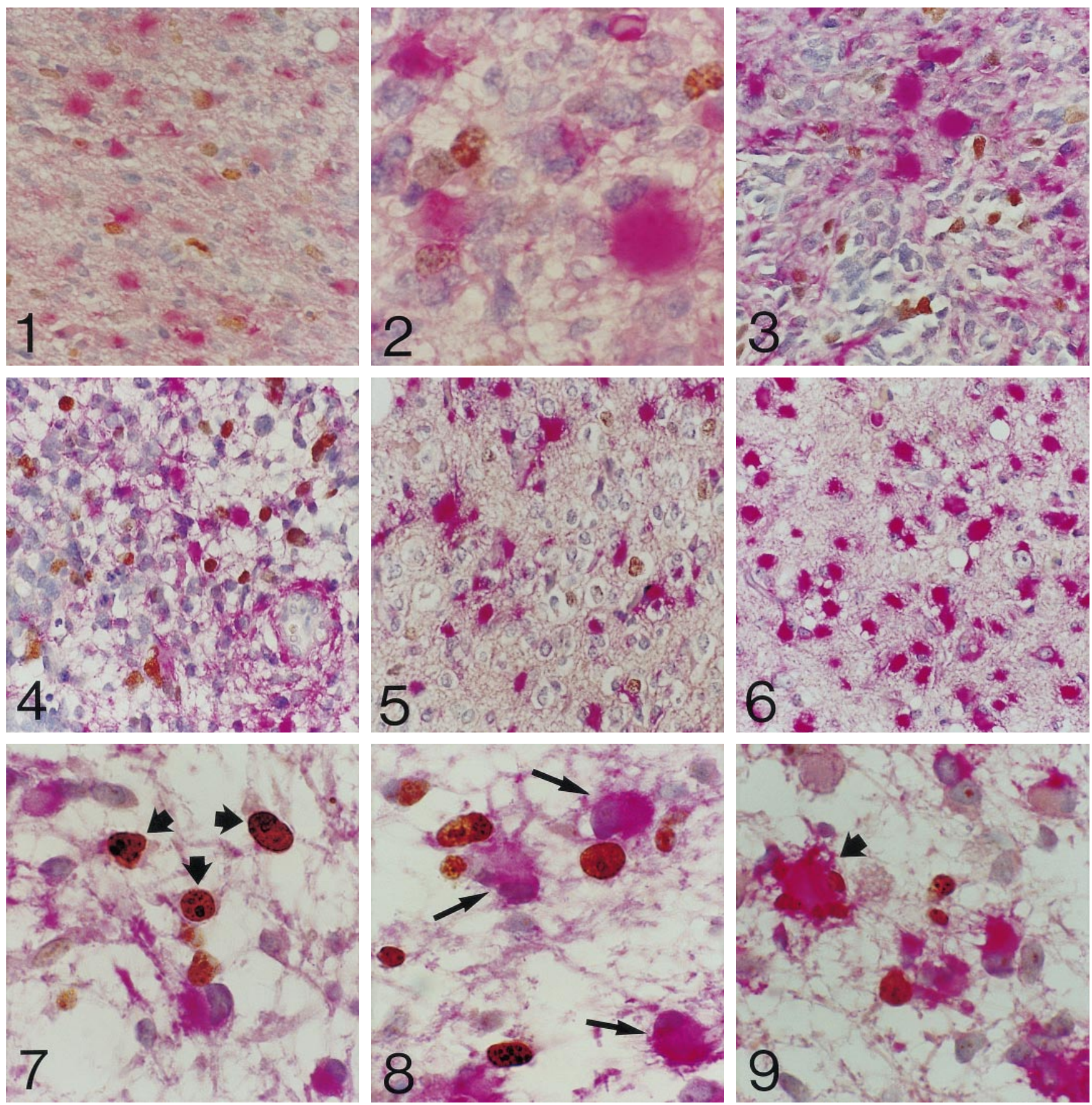

Fig. 8 Minigemistocytes (arrows) and GFOCs in an oligodendroglioma (case 31). None of the gemistocytic cells show proliferative activity, while MIB-1 immunoreactivity in nuclei of neoplastic oligodendrocytes is occasionally present. GFAP/MIB-1 double labeling, $\times 1,000$

Fig. 9 Monstrous cell (arrow) in a monstrocellular glioblastoma with positive MIB-1 staining in its nuclei (case 19). GFAP/MIB-1 double labeling, $\times 400$

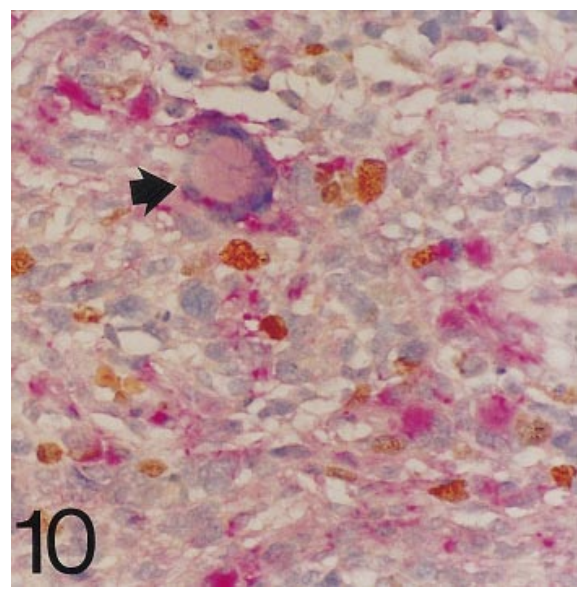

Fig. 10 In contrast to the monstrous cell in Fig. 9, this multinuclear giant cell (arrow) did not stain for MIB-1 (case 20). GFAP/ MIB-1 double labeling, $\times 150$ 
PBS, MIB-1 antibody (Immunotech, Marseille, France; mouse monoclonal, diluted 1:100 in PBS) was incubated for $2 \mathrm{~h}$ at RT. After rinsing in PBS, the sections were subsequently incubated with unconjugated goat anti-mouse antibody (Dako, The Netherlands), diluted 1:50 in PBS for $30 \mathrm{~min}$, mouse PAP complex (Sigma), diluted 1:300 in PBS for $30 \mathrm{~min}$ and 3,3'-diaminobenzidine tetrahydrochloride (Fluka, Germany) for $7 \mathrm{~min}$ at RT. Between each incubation step the sections were rinsed three times in PBS. Finally, a short counterstaining with hematoxylin was performed. Adjacent slides of each tumor were immunostained following the MIB-1 protocol only.

\section{Scoring MIB-1 LI}

The MIB-1 positive nuclei were counted in five selected fields of highest cell density, and the MIB-1 labeling index (LI) was established by calculating the quotient of positive nuclei by total number of nuclei.

\section{Results}

Large gemistocytic cells and minigemistocytes were found in astrocytic and oligodendroglial tumors (Table 1). The GFOCs were only seen in the oligodendroglial and mixed neoplasms. The numbers of these different cell types found in each glioma were estimated (Table 1). The cytoplasm of all astrocytic tumor cells and all gemistocytic cells stained positively for GFAP. The minigemistocytes and classic gemistocytes were easily detected by their ample GFAP-positive cytoplasm, while GFOCs were recognized by a small perinuclear GFAP-positive cytoplasmic rim.

MIB-1-immunoreactive cells showed a granular nuclear staining pattern. Similar percentages of MIB-1-labeled nuclei were found in sections only immunostained for MIB-1 as compared to corresponding sections that were double labeled with MIB-1 and GFAP. No cytoplasmic staining was seen. MIB-1-positive nuclei were found in all tumors and were almost absent in the cases of reactive gliosis. There were large regional differences with respect to the MIB-1 immunoreactivity within the same tumor samples (Fig. 1). Neoplastic astrocytes and oligodendrocytes often had positive nuclei (Figs. 1-10). The percentages of MIB-1-positive nuclei were highest in the glioblastomas and lowest in the gliomas of lowest malignancy grades (Table 1). Small anaplastic glial cells showed the highest labeling index (Figs. 3, 10).

None of the gemistocytic cells showed positive nuclear immunostaining (Figs. 1-10). The high labeling indices of gemistocytic astrocytomas are a result of MIB-1 labeling of the small anaplastic cells that were interspersed between the MIB-1-negative gemistocytes (Table 1; Figs. 1, 2). Nuclei of minigemistocytes, identified by their reactivity with anti-GFAP antibody, were never MIB-1 immunostained (Figs. 5-8). Some of the GFOCs were MIB-1 positive (Fig. 7). Nuclei of giant and monstrous cells showed variable immunostaining, ranging from entirely negative to occasionally positive (Table 1; Figs. 9, 10).

\section{Discussion}

Gemistocytic cells are well known to appear in a variety of reactive conditions including hypoxia or ischemia. Gemistocytes found in glial neoplasms may be interpreted as entrapped non-neoplastic glial cells that were transformed into the gemistocytic phenotype due to deprivation of nutrients caused by the increasing demands of surrounding tumor cells [8]. Alternatively, if regarded as a true tumor cell, the taxonomy of the gemistocyte is uncertain. On the one hand, gemistocytes seem to be related to the non-proliferating monstrous and giant cells residing in some high-grade gliomas [18]. On the other hand, a kinship between gemistocytes and transitional cells (i.e., minigemistocytes and GFOCs) has been hypothesized [7]. It may be that, similar to neoplastic astrocytes, neoplastic oligodendrocytes also metamorphose into large gemistocytes, possibly via the phenotypes of GFOCs and minigemistocytes. Whereas no differences in biological behavior of oligodendroglial tumors with or without transitional cells were found, survival times of patients were negatively affected by the presence of large gemistocytes in tumor samples [10]. However, the finding of negative immunostaining for MIB-1 of gemistocytes proves that correlation of the presence of these cells with short survival is not due to proliferation of gemistocytes themselves, but rather to unknown factors associated with their presence.

Although regional differences in histopathology of glial neoplasms have been the subject of various studies $[1,2,4,6,19,20]$, only a few papers concerning cell proliferation in gliomas specifically address subsets of glial cells such as gemistocytes. In early in vivo thymidine-labeling studies, it was shown that incorporation in gemistocytes was only found in autopsy specimens acquired after variable survival times. Therefore, a gradual transformation of fibrillary and protoplasmic astrocytes into gemistocytic cells was suggested [8]. In subsequent in vivo studies on cell proliferation using bromodeoxyuridine analogues no overall differences in labeling between tumors with or without gemistocytes were shown [14]. The present results, however, illustrate that the high MIB$1 \mathrm{LI}$ in gemistocytic astrocytomas is a result of immunostaining of nuclei of cells other than the gemistocytes, a finding that was recently confirmed by a comparative study on intratumoral differences in cell proliferation using an incorporation technique in addition to immunohistochemistry [19].

Classic gemistocytes are considered as inert cells appearing relatively late in tumor development $[7,8,10$, 13]. The present results underline the lack of proliferative capacity of both gemistocytes and minigemistocytes. The acquisition of GFAP-positive intermediate filaments of neoplastic oligodendrocytes might be the first step towards the non-proliferative gemistocytic cell pool, explaining diminished immunostaining for MIB-1 of the GFOCs as compared to their GFAP-negative counterparts. While incorporation techniques eventually show a meta- 
morphosis of one cell type into another, unfortunately no conclusions concerning putative transformation of glial phenotypes are possible, based on immunohistochemical studies.

Recently, the expression of fibroblast growth factor (FGF) has been specifically linked to gemistocytic cells $[15,21,25]$. Both acidic and basic FGF are angiogenic factors capable of stimulating proliferation and motility of endothelial cells, and are mitogenic for endothelial and glial cells. Acidic FGF was abundantly present in the cytoplasm of protoplasmic and gemistocytic astrocytes in particular, while contradictory results as to the cellular subsets expressing basic FGF were reported [21, 25]. It has been speculated that large quantities of FGF liberated from less-viable glial cells like gemistocytes could induce proliferation of adjacent vascular and glial tumor cells [21]. Further investigations on the characterization of patterns of expression of growth factors and their receptors might further specify the role of gemistocytic cells in the biological behavior of glial neoplasms.

\section{References}

1. Burger PC, Kleihues P (1989) Cytologic composition of the untreated glioblastoma multiforme: a postmortem study of eighteen cases. Cancer 63: 2014-2023

2. Coons WS, Johnson PC (1993) Regional heterogeneity in the proliferative activity of human gliomas as measured by the Ki67 labelling index. J Neuropathol Exp Neurol 52: 609-618

3. Daumas-Duport C, Scheithauer BW, O'Fallon J, Kelly P (1988) Grading of astrocytomas. A simple and reproducible method. Cancer 62: 2152-2165

4. Deckert M, Reifenberger G, Wechsler W (1989) Determination of the proliferative potential of human brain tumors using the monoclonal antibody Ki-67. J Cancer Res Clin Oncol 115: 179-188

5. Elvidge AR, Martinez-Coll A (1956) Long-term follow-up of 106 cases of astrocytoma, 1928-1939. J Neurosurg 13: 318331

6. Germano IM, Ito M, Cho KG (1989) Correlation of histopathological features and proliferative potential of gliomas. J Neurosurg 701-706

7. Herpers MJHM, Budka H (1984) Glial fibrillary acidic protein (GFAP) in oligodendroglial tumors. Gliofibrillary oligodendroglioma and transitional oligoastrocytoma as subtypes of oligodendroglioma. Acta Neuropathol (Berl) 64: 265-272

8. Hoshino T, Wilson CB, Ellis WG (1975) Gemistocytic astrocytes in gliomas. An autoradiographic study. J Neuropathol Exp Neurol 34: 263-281

9. Johnson H, Haymaker WE, Rubini JR, et al (1960) An autoradiographic study of a human brain and glioblastoma multiforme after the in vivo uptake of tritiated thymidine. Cancer 13 636-642
10. Kros JM, Eden CG van, Stefanko SZ, Waayer-van Batenburg M, Kwast TH van der (1990) Prognostic implications of glial fibrillary acidic protein containing cell types in oligodendrogliomas. Cancer 66: 1204-1212

11. Kros JM, Stefanko SZ, deJong AA, Vroonhoven CC van, VanderHeul RO, Kwast TH vander (1991) Ultrastructural and immunohistochemical segregation of gemistocytic subsets. Hum Pathol 22: 33-40

12. Kros JM, deJong AA, Kwast TH van der (1992) Ultrastructural characterization of transitional cells in oligodendrogliomas. J Neuropathol Exp Neurol 51: 186-193

13. Krouwer HGJ, Davis RL, Silver P, Prados M (1991) Gemistocytic astrocytomas: a reappraisal. J Neurosurg 74: 399-406

14. Labrousse F, Daumas-Duport C, Batorski L, Hoshino T (1991) Histological grading and bromodeoxyuridine labeling index of astrocytomas. Comparative study in a series of 60 cases. J Neurosurg 75: 202-205

15. Mapstone TB (1991) Expression of platelet-derived growth factor and transforming growth factor and their correlation with cellular morphology in glial tumors. J Neurosurg 75: 447-451

16. Meneses ACO, Kepes JJ, Sternberger NH (1982) Astrocytic differentiation of neoplastic oligodendrocytes. J Neuropathol Exp Neurol 41: 368

17. Nissl F (1904) Zur Histopathologie der paralytischen Rindenerkrankung. Histol Histopathol Arb Grosshirn 1: 315

18. Ogashiwa M, Nakadai M, Asoh Y, et al (1987) Astrocytic glioma: morphologic analysis of recurrent gliomas. Giant cell and gemistocytic cell formation. Neurol Med Chir (Tokyo) 27: 276-282

19. Onda K, Davis RL, Wilson CB, Hoshino T (1994) Regional differences in bromodeoxyuridine uptake, expression of Ki-67 protein, and nucleolar organizer region counts in glioblastoma multiforme. Acta Neuropathol 87: 586-593

20. Paulus W, Peiffer J (1989) Intratumoral histologic heterogeneity of gliomas. Cancer 64: 442-447

21.Paulus W, Grothe C, Sensenbrenner M, Janet T, Baur I, Graf M, Roggendorf W (1990) Localization of basic fibroblast growth factor, a mitogen and angiogenic factor, in human brain tumors. Acta Neuropathol 79: 418-423

22. Russell DS, Rubinstein LJ (1989) Pathology of tumours of the nervous system, 5th edn. Arnold, London

23. Shi S, Key M, Kalra K (1991) Antigen retrieval in formalinfixed, paraffin-embedded tissues: an enhancement method for immunohistochemical staining based on microwave oven heating of tissue sections: J Histochem Cytochem 39: 741-748

24. Smith MT, Ludwig CL, Godfrey AD, Armbrustmacher VW (1983) Grading of oligodendrogliomas. Cancer 52: 2107-2114 25. Stefanik DF, Rizkalla LR, Soi A, Goldblatt A, Rizkalla WM (1991) Acidic and basic fibroblast growth factors are present in glioblastoma multiforme. Cancer Res 51: 5760-5765

26. VanderMeulen JDM, Houthoff HJ, Ebels EJ (1978) Glial fibrillary acidic protein in human gliomas. Neuropathol Appl Neurobiol 4: 177-190

27. Wilkinson IMS, Anderson JR, Holmes AE (1987) Oligodendroglioma: an analysis of 42 cases. J Neurol Neurosurg Psychiatry 50: $304-312$ 